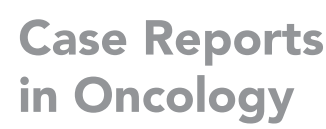

\title{
A Case of Pulmonary Tumor Thrombotic Microangiopathy Suggested by the Presence of Tumor Cells in Peripheral Blood
}

\author{
Yusuke Kawanaka ${ }^{a}$ Hisato Kawakami $^{a}$ Shigeki Shimizu ${ }^{b}$ \\ Takeshi Yoshida $^{a}$ Hidetoshi Hayashi $^{a}$ Kazuto Nishio $^{c}$ Takao Satou $^{b}$ \\ Kazuhiko Nakagawa ${ }^{a}$ \\ aDepartment of Medical Oncology, Kindai University Faculty of Medicine, Osaka-Sayama, \\ Japan; bepartment of Pathology, Kindai University Faculty of Medicine, Osaka-Sayama, \\ Japan; 'Department of Genome Biology, Kindai University Faculty of Medicine, \\ Osaka-Sayama, Japan
}

\section{Keywords}

Pulmonary tumor thrombotic microangiopathy · Trafermin - Fibroblast growth factor 2 .

Fibroblast growth factor receptor · Platelet-derived growth factor

\begin{abstract}
Pulmonary tumor thrombotic microangiopathy (PTTM) is characterized by tumor cell microemboli with occlusive fibrointimal remodeling in small pulmonary vessels. Platelet-derived growth factor (PDGF) has been implicated in the development of PTTM, and fibroblast growth factor (FGF) promotes PDGF signaling via PDGF receptor $\beta$. We here describe a cancer patient who presented with dyspnea of uncertain etiology and whose condition worsened rapidly. $A$ 68-year-old man with hypopharyngeal squamous cell carcinoma (cT4aN2bM0, stage IVA) was treated with surgery followed by radiation. Two years later, a lung metastatic lesion was surgically removed on the basis of suspected primary lung cancer. The patient was thereafter monitored without chemotherapy. Two months later, he had third-degree burns and received conservative therapy including debridement and application of trafermin (FGF2) spray. Two weeks later, he was hospitalized with complaints of fever and dyspnea. Pneumonia and pulmonary embolism were ruled out by chest computed tomography with pulmonary arteriography, whereas intravascular lymphoma was excluded by laboratory testing. Malignant cells were detected in his peripheral blood on hospital day 8 , and their number increased gradually thereafter. His respiratory symptoms worsened, and the patient died on hospital day 10. We concluded that the cause of death was PTTM, with the clinical course suggesting a possible relation to trafermin. This suggestion was supported by the detection of FGF receptor 2 overexpression in the primary tumor by immunostaining.




\section{Introduction}

When a patient with a malignancy presents with dyspnea, various diagnoses either associated with the malignancy or not are possible. Such possibilities include pulmonary embolism, intravascular lymphoma (IVL), carcinomatous lymphangiomatosis, pleural effusion, pneumonia, and drug-induced lung injury. Pulmonary tumor thrombotic microangiopathy (PTTM) is a relatively rare complication of metastatic cancer that can lead to progressive hypoxia and pulmonary hypertension with associated dyspnea. PTTM is characterized pathologically by diffuse tumor emboli and fibrocellular intimal proliferation in small pulmonary arteries and arterioles [1]. Cytokines including tissue factor, platelet-derived growth factor (PDGF), and vascular endothelial growth factor are key drivers of the development of pulmonary hypertension and hypoxia in PTTM [2]. Trafermin is a recombinant form of human fibroblast growth factor 2, which promotes signaling by PDGF by increasing the expression of PDGF receptor $\beta$ (PDGFR $\beta$ ) in pericytes [3]. We here report a case of PTTM development in a cancer patient after application of trafermin spray for treatment of burns on both feet.

\section{Case Report}

A 68-year-old man with hypopharyngeal squamous cell carcinoma (cT4aN2bM0, stage IVA) underwent pharyngeal laryngectomy and total thyroidectomy, followed by radiation therapy with a total of 66 Gy (2 Gy per day for 33 days). Two years later, computed tomography (CT) imaging showed a mass lesion in the right lung. On the basis of a suspected diagnosis of primary lung cancer, a thoracoscopic partial right pneumonectomy was performed. Pathological examination of the resected tissue revealed lung metastasis of the hypopharyngeal cancer, and the patient was monitored without chemotherapy.

Two months later, the patient had third-degree burns on both feet. Conservative therapy including debridement followed by spray application of trafermin (Fiblast Spray, Kaken, Japan) was administered. After 2 weeks, the patient was brought to the emergency department with complaints of fever and dyspnea. His oxygen saturation was $93 \%$ on receiving oxygen at $4 \mathrm{~L} / \mathrm{min}$, but his lungs were clear on auscultation. He was in shock on arrival and was hospitalized. Laboratory tests revealed a blood urea nitrogen level of $79 \mathrm{mg} / \mathrm{dL}$, creatinine of 3.14 $\mathrm{mg} / \mathrm{dL}, \mathrm{C}$-reactive protein of $19 \mathrm{mg} / \mathrm{dL}$, lactate dehydrogenase of $6749 \mathrm{U} / \mathrm{L}$, and D-dimer of $9.1 \mu \mathrm{g} / \mathrm{mL}$. CT imaging did not reveal evidence of metastatic tumors, infectious pneumonia, or other abnormalities. Treatment with the broad-spectrum antibacterial agent meropenem was initiated based on a suspicion of wound infection. On his second day in hospital, the dyspnea gradually worsened, with his oxygen demand increasing from 4 to $10 \mathrm{~L} / \mathrm{min}$ over $24 \mathrm{~h}$. Chest CT with pulmonary arteriography (CTPA) revealed no abnormality other than a small amount of pleural effusion. We measured the serum level of soluble interleukin-2 receptor (sIL-2R) in order to assess the possibility of IVL, but the value was not especially high $(669 \mathrm{U} / \mathrm{mL})$, suggesting against this possibility. Kidney function gradually worsened, resulting in hyperkalemia $(7.7 \mathrm{mEq} / \mathrm{L})$. On hospital day 8, malignant tumor cells were detected in peripheral blood (Fig. 1), and their number began to increase thereafter. Given that neither myeloblast nor lymphoblast cells were detected, a diagnosis of bone marrow carcinosis was ruled out. Even with full medical support, multiple organ failure progressed rapidly, and the patient died on hospital day 10.

The malignant tumor cells in peripheral blood were positive for p40, a marker of squamous cell carcinoma, suggestive of recurrence of the patient's hypopharyngeal cancer without any radiographic change. We therefore concluded that the cause of death was PTTM. To investigate the possibility that the trafermin (FGF2) spray might have caused PTTM, we

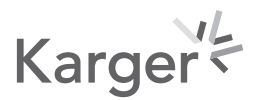


Fig. 1. Malignant tumor cells which were detected in peripheral blood.

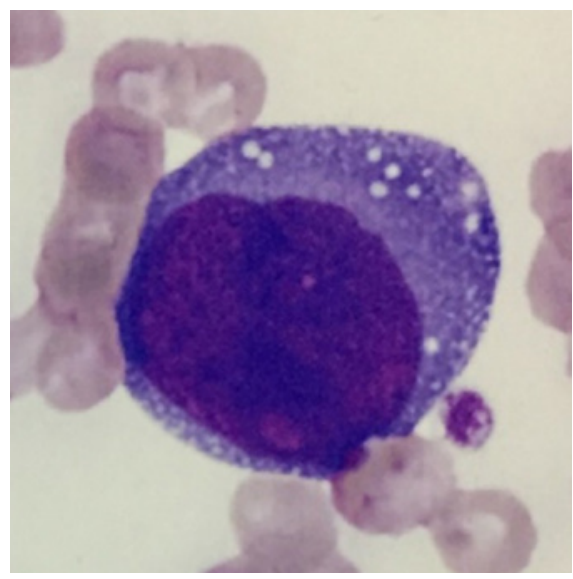

performed immunostaining for fibroblast growth factor receptor 2 (FGFR2) - the receptor largely responsible for mediating FGF2-induced pericyte recruitment in tumors [3] - as well as droplet digital polymerase chain reaction (ddPCR) analysis for the FGFR2 gene in archived primary tumor tissue. Immunostaining revealed FGFR2 overexpression, whereas ddPCR revealed no genetic alteration including amplification or point mutation of FGFR2.

\section{Discussion}

For cancer patients with rapid respiratory failure, possible diagnoses include pulmonary embolism, IVL, and PTTM among others. A diagnosis of pulmonary embolism is usually based on elevation of the circulating D-dimer level, certification of right ventricle strain by echocardiography, and detection of a thrombus in a pulmonary artery by CTPA [4]. In the present case, although the D-dimer level had increased to $9.1 \mu \mathrm{g} / \mathrm{mL}$, CTPA did not detect a thrombus in a pulmonary artery, thus precluding a diagnosis of pulmonary embolism. A diagnosis of IVL is based on the identification of lymphoma cells within small- to medium-sized blood vessels. A biopsy of involved skin, senile hemangioma, or random biopsy of apparently normal skin may be diagnostic [5]. An abnormally high level of sIL-2R $(>5,000 \mathrm{U} / \mathrm{mL})$ is usually also observed [6]. However, the level of sIL-2R in the proband $(669 \mathrm{U} / \mathrm{mL})$ did not achieve such a threshold, thus indicating against the possibility of IVL, although skin biopsy was not performed.

The diagnosis of PTTM can be challenging and depends on many findings that are nonspecific. Laboratory tests, echocardiography, pulmonary function tests, radionucleotide ventilation perfusion scanning, CT imaging, pulmonary wedge aspiration cytology, and lung biopsy are suggested to allow such a diagnosis. Elevated fibrinogen degradation product or D-dimer levels have been a uniform finding in PTTM [2]. Pulmonary wedge aspiration cytology has been found to have a sensitivity and specificity of $80-88 \%$ and $82-94 \%$, respectively, for the diagnosis of PTTM [7]. Lung biopsy has been applied to diagnose PTTM antemortem, either guided by CT, bronchoscopically, or with the use of video-assisted thoracoscopic surgery [7-9].

In the present case, the number of malignant cells in peripheral blood began to increase gradually, despite the absence of signs of cancer recurrence on CT. Bone marrow carcinosis can give rise to the presence of malignant tumor cells in peripheral blood, but neither myeloblast nor lymphoblast cells were detected in peripheral blood of the present patient, thus denying such a diagnosis. As far as we are aware, this is the first report of a case of suspected 
PTTM associated with the detection of malignant cells in peripheral blood. Our findings may thus be of help in making such a diagnosis for patients with malignancy who present with dyspnea of uncertain etiology.

The term PTTM was coined by von Herbay et al. [1] in 1990, with the disease process being suggested to be characterized by embolization of pulmonary arterioles by tumor cells followed by activation of the coagulation cascade, thrombus formation, and fibrocellular intimal proliferation. Cytokines including tissue factor, PDGF, and vascular endothelial growth factor are key drivers of the development of pulmonary hypertension and hypoxia in PTTM [2]. What triggered the rapid emergence of PTTM in the present case remains unknown, but we speculate that topical trafermin (FGF2) may have been responsible. FGF2 is thus known as a tumor growth factor [3], and the malignant cells in the peripheral blood of the proband were squamous cell carcinoma, suggesting that they reflected recurrence of his primary tumor. Immunostaining and ddPCR analysis of primary tumor tissue revealed FGFR2 overexpression but no genetic alteration of FGFR2 in the tumor cells, suggesting the possibility of innate FGF2 dependency of the tumor. FGF2 is also a potent pericyte-stimulating factor in tumors [3]. FGF2 binding to FGFR2 promotes PDGF-PDGFR $\beta$ signaling and thereby stimulates pericyte proliferation and recruitment. Perivascular cells are important cellular components of the tumor microenvironment, and they modulate vascular integrity, remodeling, stability, and function [3]. Abnormal pericyte proliferation and recruitment as a result of activation of PDGF-PDGFR $\beta$ signaling can trigger a thrombogenic cascade on the surface of tumor embolisms and the proliferation of vascular cells [2]. Of note, PTTM has been associated mostly with gastric cancer, in which the frequency of FGFR2 overexpression is higher than that in most other malignancies $[10,11]$.

In summary, we report here the case of a cancer patient with dyspnea of uncertain etiology whose condition deteriorated rapidly. Although a full examination was not performed, the clinical course of the patient as well as the appearance of malignant tumor cells in peripheral blood suggested PTTM as the cause of death. Exploratory analysis also implicated trafermin as a possible trigger for PTTM development, a possibility that warrants further study.

\section{Acknowledgement}

The authors would like to acknowledge the Pathology Institute Corp. for pathological support.

\section{Statement of Ethics}

The patient's family has given their written informed consent to publish his case (including publication of images).

\section{Conflict of Interest Statement}

H.K. has received consulting fees from Bristol-Myers Squibb Co. Ltd., Eli Lilly Japan K.K., MSD K.K., Ono Pharmaceutical Co. Ltd., Daiichi-Sankyo Co. Ltd., and Taiho Pharmaceutical Co. Ltd.; honoraria from Bristol-Myers Squibb Co. Ltd., AstraZeneca K.K., Bayer Yakuhin Ltd., Eli Lilly Japan K.K., MSD K.K., Ono Pharmaceutical Co. Ltd., Chugai Pharmaceutical Co. Ltd., Daiichi Sankyo Co. Ltd., Takeda Pharmaceutical Co. Ltd., and Taiho Pharmaceutical Co. Ltd.; lecture 
fees from Bristol-Myers Squibb Co. Ltd., Eli Lilly Japan K.K., MSD K.K., Ono Pharmaceutical Co. Ltd., Chugai Pharmaceutical Co. Ltd., Takeda Pharmaceutical Co. Ltd., and Taiho Pharmaceutical Co., Ltd.; and research funding from Chugai Pharmaceutical Co. Ltd. and Eisai Co. Ltd.

H.H. has received honoraria from AstraZeneca K.K., Novartis Pharmaceuticals K.K., Boehringer Ingelheim Japan Inc., Bristol-Myers Squibb Co. Ltd., Chugai Pharmaceutical Co. Ltd., Eli Lilly Japan K.K., MSD K.K., Ono Pharmaceutical Co. Ltd., Pfizer Japan Inc., and Taiho Pharmaceutical Co. Ltd. as well as research funding from AstraZeneca K.K., Boehringer Ingelheim Japan Inc., Bristol-Myers Squibb Co. Ltd., and Ono Pharmaceutical Co. Ltd.

T.Y. has received honoraria from Daiichi-Sankyo Co. Ltd., Hisamitsu Pharmaceutical (China) Co., Ltd., Mundipharma Pharmaceutical Co. Ltd., Kyowa Pharmaceutical Industry Co., Ltd.

K.N. has received honoraria from AstraZeneca K.K., Astellas Pharma Inc., MSD K.K., Ono Pharmaceutical Co. Ltd., Nippon Boehringer Ingelheim Co. Ltd., Novartis Pharma K.K., Pfizer Japan Inc., Clinical Trial Co. Ltd., Medicus Shuppan Publishers Co. Ltd., Care Net Inc., Reno Medical K.K., Kyorin Pharmaceutical Co. Ltd., Medical Review Co. Ltd., Roche Diagnostics K.K., Bayer Yakuhin Ltd., Medical Mobile Communications Co. Ltd., Nichi-Iko Pharmaceutical Co. Ltd., Takeda Pharmaceutical Co. Ltd., Taiho Pharmaceutical Co. Ltd., Bristol Myers Squibb Co. Ltd., Eli Lilly Japan K.K., SymBio Pharmaceuticals Ltd., Chugai Pharmaceutical Co. Ltd., Nanzando Co. Ltd., Nikkei Business Publications Inc., Daiichi Sankyo Co. Ltd., Thermo Fisher Scientific K.K., Yomiuri Telecasting Corp., Nippon Kayaku Co. Ltd., Merck Biopharma Co. Ltd., and AbbVie Inc. as well as research funding from MSD K.K., inVentiv Health Japan, Daiichi Sankyo Co. Ltd., AbbVie Inc., Icon Japan K.K., Takeda Pharmaceutical Co. Ltd., Gritsone Oncology Inc., Eli Lilly Japan K.K., Bristol Myers Squibb Co. Ltd., Taiho Pharmaceutical Co. Ltd., Parexel International Corp., Ono Pharmaceutical Co. Ltd., AstraZeneca K.K., Kissei Pharmaceutical Co. Ltd., EPS Corp., Syneos Health, Pfizer R\&D Japan G.K., A2 Healthcare Corp., Astellas Pharma Inc., Novartis Pharma K.K., Quintiles Inc./IQVIA Services Japan K.K., Chugai Pharmaceutical Co. Ltd., EP-CRSU Co. Ltd., Linical Co. Ltd., Eisai Co. Ltd., Nippon Boehringer Ingelheim Co. Ltd., Pfizer Japan Inc., SymBio Pharmaceuticals Ltd., Merck Serono Co. Ltd./Merck Biopharma Co. Ltd., CMIC Shift Zero K.K., Kyowa Hakko Kirin Co. Ltd., Bayer Yakuhin Ltd., EPS International Co. Ltd., and Otsuka Pharmaceutical Co. Ltd.

\section{Funding Sources}

No financial support was used for this case report.

\section{Author Contributions}

Y. Kawanaka searched the literature and wrote the manuscript. H. Kawakami conceived and edited the manuscript. T. Yoshida treated the patient. S. Shimizu and T. Satou gave us input about pathology. K. Nishio performed ddPCR analysis. All authors have read and approved the final manuscript. 


\section{References}

1 von Herbay A, Illes A, Waldherr R, Otto HF. Pulmonary tumor thrombotic microangiopathy with pulmonary hypertension. Cancer. 1990 Aug 1;66(3):587-92.

2 Price LC, Wells AU, Wort SJ. Pulmonary tumour thrombotic microangiopathy. Curr Opin Pulm Med. 2016 Sep; 22(5):421-8.

3 Hosaka K, Yang Y, Nakamura M, Andersson P, Yang X, Zhang Y, et al. Dual roles of endothelial FGF-2-FGFR1PDGF-BB and perivascular FGF-2-FGFR2-PDGFR $\beta$ signaling pathways in tumor vascular remodeling. Cell Discov. 2018;4:3.

4 Righini M, Robert-Ebadi H, Le Gal G. Diagnosis of acute Pulmonary Embolism. J Thromb Haemost. 2018 Feb; 15(7):1251-61.

5 Ponzoni M, Ferreri AJ, Campo E, Facchetti F, Mazzucchelli L, Yoshino T, et al. Definition, diagnosis, and management of intravascular large B-cell lymphoma: proposals and perspectives from an international consensus meeting. J Clin Oncol. 2007 Jul 20;25(21):3168-73.

6 Shimada K, Matsue K, Yamamoto K, Murase T, Ichikawa N, Okamoto M, et al. Retrospective analysis of intravascular large B-cell lymphoma treated with rituximab-containing chemotherapy as reported by the IVL study group in Japan. J Clin Oncol. 2008 Jul 1;26(19):3189-95.

7 Kane RD, Hawkins HK, Miller JA, Noce PS. Microscopic pulmonary tumor emboli associated with dyspnea. Cancer. 1975 Oct;36(4):1473-82.

8 Miyano S, Izumi S, Takeda Y, Tokuhara M, Mochizuki M, Matsubara O, et al. Pulmonary tumor thrombotic microangiopathy. J Clin Oncol. 2007 Feb 10;25(5):597-9.

9 Kayatani H, Matsuo K, Ueda Y, Matsushita M, Fujiwara K, Yonei T, et al. Pulmonary tumor thrombotic microangiopathy diagnosed antemortem and treated with combination chemotherapy. Intern Med. 2012;51(19): 2767-70.

10 Kitamura A, Nishimura N, Jinta T, Suda R, Yamano Y, Ishikawa G, et al. A case of pulmonary tumor thrombotic microangiopathy diagnosed by transbronchial lung biopsy and treated with chemotherapy and long-term oxygen and anticoagulation therapies. Case Rep Pulmonol. 2013;2013:259080.

11 Ogawa A, Yamadori I, Matsubara O, Matsubara H. Pulmonary tumor thrombotic microangiopathy with circulatory failure treated with imatinib. Intern Med. 2013;52(17):1927-30. 